\title{
La acción tutorial en el posgrado Reflexiones y principales desafíos en la formación investigativa
}

\section{Tutorial action at the graduate level Reflections and main challenges in research training.}

\author{
DOI: $10.46932 / \mathrm{sfjdv2n4-054}$
}

Received in: March 1st, 2021

Accepted in: May 30th, 2021

\author{
Elia Olea Deserti \\ Doctora en Filosofía y Ciencias de la Educación \\ Instituto Politécnico Nacional. ESCA Unidad Santo Tomás \\ Manuel Carpio 471, Plutarco Elías Calles, 11340, Ciudad de México \\ E-mail: eliaolea@gmail.com, eolead@ipn.mx \\ Ruth Salazar Pulido \\ Maestría en Administración, Gestión y Desarrollo de la Educación \\ Benemérita Escuela Nacional de Maestros \\ Calz México-Tacuba 75, Un Hogar Para Nosotros, 11330, Ciudad de México \\ E-mail: rusa0910@yahoo.com.mx
}

\section{RESUMEN}

En el momento actual, siglo XXI, es muy importante hacer una reflexión sobre la acción tutorial que se ofrece en el posgrado a los estudiantes de maestría y doctorado. Para ello, es necesario hacer un análisis del contexto, la normativa, los aspectos inherentes a la acción investigativa en cuanto a los saberes y las acciones y se presenta un panorama general de las características de los tutores y tutorados. Antes de la discusión y conclusiones, se incluyen los principales desafíos que, a juicio de las autoras, se tienen que atender y dar respuesta ya que el avance de un país depende fundamentalmente de la investigación, el desarrollo y la innovación realizada por los investigadores, quienes son formados en las secciones de posgrado der las instituciones educativas, con el acompañamiento, el apoyo y la orientación de un académico-tutor.

Palabras clave: Tutoría posgrado, Formación investigativa posgrado, Desafíos tutoría posgrado.

\begin{abstract}
At the present time, XXI century, it is very important to make a reflection on the tutorial action offered to master and doctoral students at the postgraduate level. For this purpose, it is necessary to make an analysis of the context, the regulations, the aspects inherent to the research action in terms of knowledge and actions, and an overview of the characteristics of tutors and tutored students is presented. Before the discussion and conclusions, the main challenges that, in the authors' opinion, must be addressed and responded to are included, since the progress of a country depends fundamentally on the research, development and innovation carried out by researchers, who are trained in the postgraduate sections of the educational institutions, with the accompaniment, support and guidance of an academic-tutor.
\end{abstract}

Key words: Postgraduate tutoring, Postgraduate research training, Postgraduate tutoring challenges. 


\section{INTRODUCCIÓN}

La segunda década del siglo XXI se orienta al desarrollo, la innovación y la formación del capital humano integral que respondan al contexto, lo que ha propiciado el aumento del interés de formar investigadores que desde muy jóvenes cuenten con las características que ayuden al desarrollo científico y tecnológico del país.

A través de las innovaciones, productos de investigación en las universidades o en las empresas, desarrolladas por sus exestudiantes de posgrados (egresados de maestrías en ciencias y doctorados), el sector productivo (de bienes y servicios) consigue ventajas competitivas. Así que de acuerdo a algunos investigadores, ha empezado a ser de uso general la "metodología contextualizada a la realidad contemporánea denominada Quinta Hélice Sistémica” (Martínez, 2012, p. 31). Es necesario que se fomente la vinculación entre la academia, con sus áreas de investigación y que como una atención a las necesidades contextuales formen a egresados pensantes que analicen e investiguen; el sector gobierno que se expresa a través de las políticas generales y las propias de cada institución educativa y que otorgan presupuesto; la comunidad local/regional que hace desarrollo; las cámaras empresariales que ayudan a solucionar las necesidades de los empleadores, que requieren de usuarios que desarrollen/innoven los productos o servicios y los consultores que conocen el know how por lo que establecen las normas de competencia a nivel nacional e internacional.

El progreso de un país gira alrededor de la formación de investigadores a través de sus universidades. En el caso de Instituto Politécnico Nacional (IPN) en sus programas educativos de posgrado, el docente académico -tutor- tiene un papel preponderante que de acuerdo al Reglamento General de Estudios define al tutor como "al personal académico asignado para acompañar, orientar y asesorar al alumno en su trayectoria escolar con la finalidad de que concluya satisfactoriamente sus estudios" (2011, p. 8). Sin embargo, poco se habla sobre los tutores a nivel posgrado, de ahí que se decidió investigar al respecto.

Objetivo de la investigación. Aportar información sobre qué repercusiones tiene la acción tutorial en la formación de investigadores nóveles.

Metodología. Es una investigación de carácter documental.

Fue necesario desarrollar este tipo de estudio en un primer momento ya que la investigación científica tiene sus raíces en la historia y en el ámbito contextual, que se tocan en los apartados. En la segunda fase, a cubrirse, se partirá de un diseño no experimental, cualitativo exploratorio en el que se obtendrá información de los tutores-directores de tesis de programas de posgrado del área de las ciencias sociales. 


\section{FORMACIÓN DE INVESTIGADORES EN LOS CENTROS EDUCATIVOS A NIVEL SUPERIOR}

\subsection{NORMATIVA}

Las instituciones educativas de nivel superior, además de ser reglamentadas por los aspectos filosóficos instituidos en el Artículo tercero de la Constitución Política, se guían por la influencia de la normativa nacional como la Ley General de Educación que en su Artículo 7, inciso VII establece la necesidad de "Fomentar actitudes que estimulen la investigación y la innovación científica y tecnológicas, así como su comprensión, aplicación y uso responsables" (fracción reformada el 19-12-2014, p. 3).

En el caso del Instituto Politécnico Nacional, la Ley Orgánica en su Artículo 4, inciso IV menciona el establecimiento y desarrollo de los medios y los procedimientos que permitan a los estudiantes incorporarse a los programas de investigación científica y tecnológica.

De acuerdo al Reglamento General de Estudios, en su Artículo 36, inciso IV, se estipula que el tutor orienta al estudiante para definir las unidades académicas y los créditos, en cada reinscripción, de tal forma que sus tutorados concluyan el programa de estudios en tiempo y forma.

El Reglamento de Estudios de Posgrado (2006) en sus Artículos 37, 38 y 41 se habla del consejero de estudios, que luego se convierte en director de tesis quien actúa como tutor ya que realiza las funciones de apoyo pedagógico, acompañamiento, orientación e incluso de apoyo socio-afectivo, en caso de ser necesario. Asimismo también realiza acciones pedagógicas en cuanto al desarrollo y escritura de la tesis, supervisando los informes que se presentan al comité tutorial.

Se consideró importante agregar, en el caso del Reglamento de Estudios de Posgrado vigente a partir del 15 de septiembre de 2017, que se reconoce a los directores de tesis como tutores, relacionados con la trayectoria escolar y el desarrollo de tesis por el maestrante y el doctorando (Artículos 18, 20, 31, 33, 35, 92, 116). En el Artículo 119, inciso II se dice que los profesores colegiados pueden "Fungir como director de tesis, miembro de comités tutoriales y tutor a nivel posgrado" (IPN, 2017, p. 56).

El Programa de Desarrollo Institucional del IPN (2015-2018), en la presentación del mismo, en el inciso 4 se habla de la necesidad de formar el capital humano calificado en las áreas de investigación básica, aplicada y desarrollo tecnológico que "permita reforzar la formación de los estudiantes politécnicos desde su ingreso al nivel medio superior, de manera que desde entonces adquieran conocimientos y capacidades para la investigación..." (2015, p. 11).

El Modelo educativo del IPN establece que para que opere el modelo, debe haber un sistema institucional de tutorías "para facilitar el seguimiento y acompañamiento de los estudiantes desde su 
ingreso hasta su egreso" (2004, p. 101). En el caso del posgrado, es un acompañamiento en el tránsito, la corresponsabilidad de un tutor y un comité tutorial en el trabajo investigativo del estudiante.

\subsection{FUNCIONES TUTORIALES}

Antes de hablar de las tutorías, se pensó conveniente mencionar que de acuerdo a la conferencia magistral de Torres (2016) presidenta del COMIE ${ }^{i}$, las prácticas científicas en el siglo XXI han producido un cambio en la producción del conocimiento por lo que se está tendiendo a consolidar los colectivos para hacer investigación a través de grupos en los que se aprenden -además de la investigación- los valores y los significados culturales. Los grupos de investigación de acuerdo a esta académica son unidades organizativas con grados de plasticidad que atienden formas funcionales y dinámicas que se transforman en el tiempo en interacciones. Su finalidad es obtener productos de investigación, formar estudiantes, obtención de grados académicos, aportar y obtener conocimiento. Tal es el caso de los grupos de posgrado en los cuales un tutor (académico) forma a un estudiante inscrito en programas de este nivel.

En el siglo actual, la educación terciaria ha sido considerada como un nivel educativo de excelencia que va a formar integralmente profesionales que efectuarán investigación y proveerán la solución a problemas y necesidades nacionales, fortalecerán el desarrollo de la ciencia y la tecnología y buscarán una vinculación con el sector productivo. Aunque existe la creencia de que en las áreas médico-biológicas y la de las ciencias duras se requiere de docentes y estudiantes que tengan una gran dedicación por ser los científicos; sin embargo en el caso de las ciencias sociales y administrativas y de la psicología/pedagogía, también se cumple con el nivel de preparación académica, disciplina y trabajo, aplicado a su ámbito propio.

Independientemente de que un programa de posgrado sea en ciencias o con orientación profesional se requiere hacer investigación con el acompañamiento del tutor quien es el docente académico graduado, con competencias comunicativas que repercuten en la motivación del estudiante ya que trabajan juntos, le apoya metodológicamente y en relación a lo teórico (contenidos), con base al objeto de estudio que es el eje que orienta el desarrollo de la investigación.

De acuerdo a Sánchez (2010) el "investigar es un saber práctico"ii; es saber hacer algo: producir conocimientos" (p. 9) lo que es propio de los alumnos de licenciatura o de posgrado (maestrías o doctorados) quienes desarrollan una tesis como un trabajo escrito, producto de una investigación a partir de un planteamiento que se pretende comprobar o de dar solución a un problema.

Cuando se quiere aprender a investigar, se hace al lado de alguien quien tiene la experiencia; es hacer y experimentar la acción por lo que hay repercusiones a mediano y largo plazos. De acuerdo a las autoras de esta ponencia, se requiere habilidad comunicativa, tanto presencial como mediante el uso de 
Tecnología de la Información y Comunicación (TIC) a través del diálogo entre el académico-tutor y el estudiante en formación, con retroalimentación permanente por parte del docente.

El tutor enseña y transmite saberes teóricos (know that) que guían el actuar del estudiante con base a la situación problemática que se aborda a través de la investigación y saberes prácticos (know how) referidas a las estrategias metodológicas que el educando va aplicando a partir del diseño de investigación, acompañado de los valores éticos (morales y epistémicos, como amor a la verdad). De hecho, el tutor tiene que guiar a los estudiantes cuyo trabajo de investigación concuerde con su línea de generación y aplicación del conocimiento (LGAC). La relación dialógica que se establece entre ambos fundamenta la enseñanza práctica "que se enseña haciendo, corrigiendo, mostrando como, haciendo repetir. Es una enseñanza práctica que no es general ni abstracta, sino con base en situaciones y problemas reales y concretos" (Sánchez, 2010, p. 28). Por tanto, los tutores deben estar actualizados y haciendo investigación y produciendo-publicando documentos relacionados con su LGAC.

El estudiante recupera los saberes prácticos como producto de su reflexión completando su saber y su saber-hacer de acuerdo a su experiencia y los problemas que haya tenido durante el desarrollo de su investigación en su tesis de grado. Hay que formar estudiantes que sean capaces de pensar como investigadores, con capacidad para realizar trabajo constante, que exista compromiso y valores éticos (Anderson en Ibarrola y Anderson, 2015).

Así que la tutoría debe ser concebida como la acción de un académico cuyas funciones son realizadas longitudinalmente y en forma transversal ya que la acción se manifiesta en las unidades de aprendizaje que el educando cursa durante los cuatro o seis semestres que tienen de duración un programa de maestría o de doctorado, además del acompañamiento permanente durante el desarrollo y conclusión de la investigación que se plasmará en su tesis. También se considera que "La tarea del tutor, entonces, consiste en estimular las capacidades y procesos de pensamiento, de toma de decisiones y de resolución de problemas (ANUIES, 2011, p. 38). El tutor atiende, orienta y está al lado de los estudiantes para apoyarlos tanto en lo cognoscitivo como lo afectivo durante su proceso de aprendizaje, al mismo tiempo para desarrollar/ejercitar su creatividad, con base en la reflexión lo que repercute en el nivel personal, institucional, social y nacional.

La calidad de los estudios de posgrado no es sólo la forma original en que se contribuye al conocimiento con una tesis, producto de una investigación, sino también trabajar "en el dominio de un amplio cuerpo de conocimientos y habilidades, así como en acercarse más a los problemas de desarrollo del país" (Díaz Barriga y Luna, 2014, p. 273). De igual forma, la investigación educativa, como en otras áreas de aplicación, "reflejan la agenda de investigación que interesa a los especialistas en el tema que 
participan de los programas de posgrado, ya sea que se trate de estudiantes o se trate de tutores" (Barrón y Gutiérrez, 2008, en Díaz Barriga y Luna, 2014, p. 284).

Cabe mencionar que los estudiantes también favorecen al tutor en cuanto a que les despiertan el interés en otros objetos de investigación, estimulando la creatividad y desarrollando su experiencia académica-investigativa en otros ámbitos y con puntos de vista -probablemente- distintos; además de que puede aumentar el gusto y la satisfacción por las acciones de sus tutorados. En la relación tutor-tutorado, ambos aprenden uno del otro.

\section{3 ¿QUIÉNES SON LOS TUTORES?}

Respecto a quién es el docente que realiza funciones de tutor, de acuerdo a El Sahili, (2011) hay características que influencian su conducta:

- Género. Se considera el aspecto biológico y el papel social que se le asigna contextualmente. Tal aspecto, combinado con la edad y el cambio hormonal, puede traer como resultado un estrés que se manifiesta más explícitamente en las féminas, quienes en forma general se responsabilizan de los hijos y el hogar.

- Edad. Período de tiempo que se ha vivido y en el que ¿se ha evolucionado?. Los docentes de posgrado -en general- tienen 54 años de edad, en promedio. Este hecho influye en sus expectativas, significado otorgado a los programas educativos y la forma en que consideran deben orientar a sus dirigidos para que aprendan a investigar. La edad influye en el nivel de apertura ante el paradigma educativo vigente, uso de las TIC, concepto de lo que es enseñar a hacer y a aprender que repercute en los saberes de sus estudiantes.

- Desarrollo humano. Durante el proceso de vida, se van desenvolviendo los aspectos de personalidad que se expresan en las actitudes y la forma de interactuar con otras personas. Se manifiesta como:

- Autoestima. De acuerdo a Flores, Vargas y Domínguez (2014) se refiere al sentimiento de aprecio que se atribuyen las personas a sí mismas que se traduce en el sentimiento de aceptación por lo que influye en el propio concepto de valía y estatus personal. Influye en la productividad y en la creatividad.

- Asertividad. Se expresa como afirmación personal, autoconfianza, autoestima y seguridad con la que se expresan los puntos de vista en el momento en que se interactúa con los demás.

- Emociones. Impulsan a la acción y cuando son muy intensas repercuten en la homeostasis corporal, sacando al cuerpo de su balance, ya sea acelerándolo o paralizándolo. Están presentes en el comportamiento, regulando las relaciones. Cuando se tienen emociones positivas respecto a 
otras personas, hay motivación a un acercamiento y las emociones negativas se traducen en evitación (Martínez, Retana y Sánchez, 2009).

- Inteligencia. Para Resing y Drenth, (2007) es el conjunto de las habilidades cognitivas o intelectuales que permiten allegarse conocimientos necesarios para resolver problemas y enfrentar desafíos. Dicha capacidad la poseen todos los docentes y los estudiantes ya que los primeros cuentan con estudios de posgrado y los alumnos tienen estudios (licenciatura) que les permiten desarrollarse en el ámbito laboral.

En cuanto a las funciones propias de la dirección-tutoría, a nivel de posgrado, para el desarrollo de su tesis, las autoras de esta comunicación las agruparon de acuerdo a las actividades presentadas en la figura 1 .

Figura 1. Funciones que realizan los tutores de posgrado

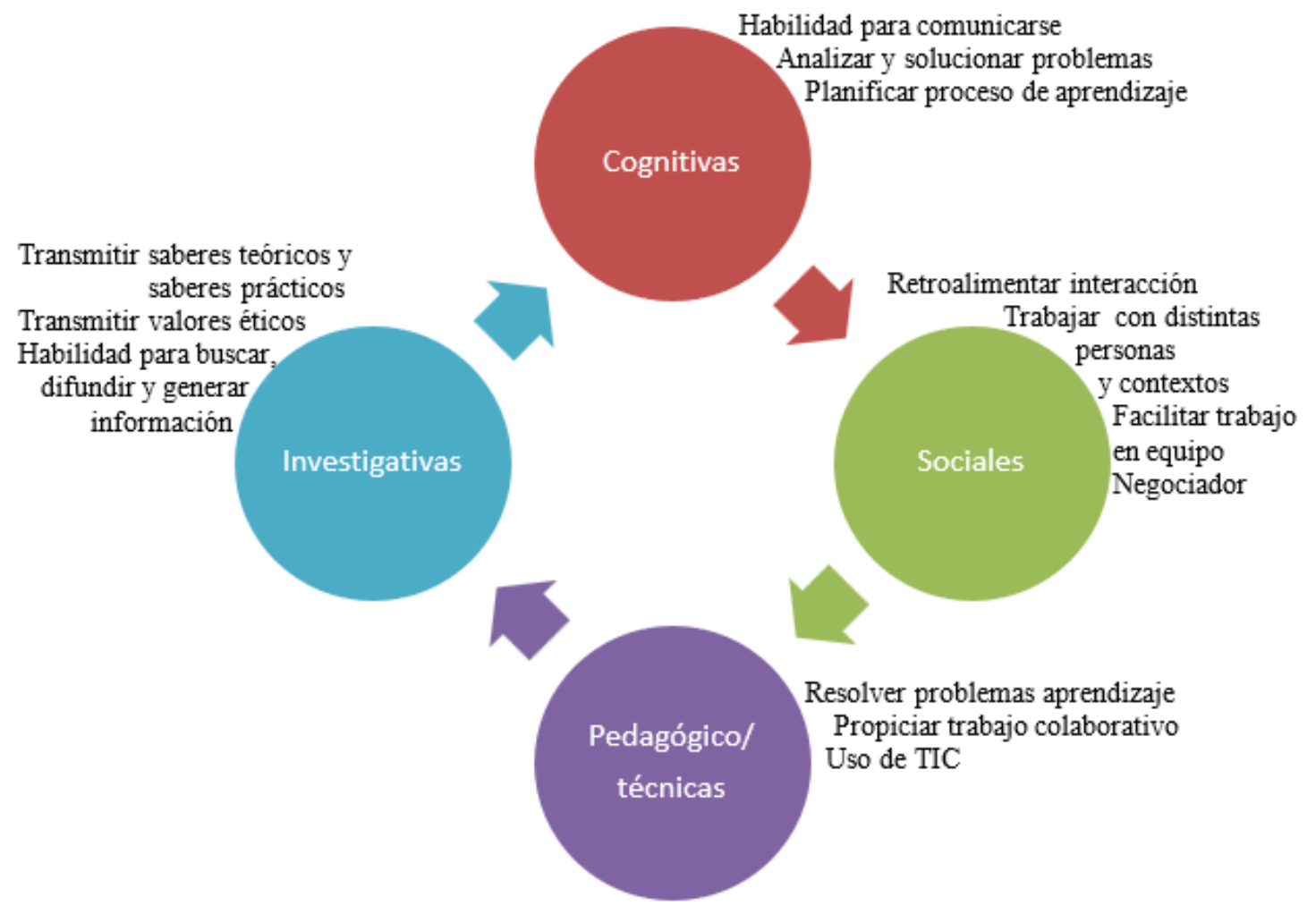

Existen también factores que pueden provocar ansiedad en los docentes (Olea, 2016) sin llegar al síndrome de burnout ${ }^{\mathrm{iii}}$ y que pueden repercutir en sus funciones tutoriales como son:

- Modificación de sus roles por cambios en el modelo pedagógico, en el tipo de currículo, en la obligatoriedad del uso de recursos tecnológicos (el tutorado esté fuera de la ciudad)

- Discrepancia en los valores o posición ideológica de los tutorados

- Disposición de recursos materiales y condiciones de trabajo 
- Política educativa y normativa institucional

- Motivación distinta entre el tutor y el tutorado respecto al trabajo de investigación

\subsection{DESAFÍOS}

El que un docente posea grado de doctor o incluso cursos postdoctorales, no garantiza que sea un buen tutor-director de tesis puesto que este académico debe ser investigador en la práctica que sepa enseñar a investigar por lo que debe manejar los saberes teóricos, prácticos y pedagógicos y llevar a cabo las funciones cognitivas, sociales, pedagógico/técnicas y las investigativas.

Los consejeros de estudios y luego directores de tesis en los programas de posgrado, tienen una formación académica propia de sus disciplinas por lo que la orientación que hacen es de acuerdo a esa óptica. Es necesario que ese docente aplique con sus dirigidos y con quienes está formando en investigación el aprender a aprender, "comprendiendo y operando sobre el saber para resolver problemas" (López, 2010, p. 109).

El que una institución cuente con investigadores connotados que sean tutores (directores de tesis de doctorandos) puede tener dificultades por falta de tiempo del tutor para enseñar los saberes teóricos y prácticos referentes a la investigación, falta de compromiso con la institución y el programa e incluso, esto puede crear problemas entre el tutor y el tutorado.

La influencia de las características de género, edad o de manejo de la emoción de parte del tutor puede reflejarse en una conducta que se identifica con dos polos, de explosiva y rígida a una muy laxa o protectora que impida el crecimiento y formación de investigadores noveles.

\section{DISCUSIÓN Y CONCLUSIONES}

De acuerdo al NMC Horizon Report: 2016 Higher Education se está transformando el paradigma de cómo funcionan las escuelas de niveles superiores. se dice que hay una desvinculación entre las demandas del siglo XXI y la manera en que se forman los graduados por lo que es necesario el contar con políticas nuevas, currículos con uso de las TIC y estudiantes impulsados a trabajar en grupos interdisciplinarios para así poder encontrar soluciones innovativas a problemas complejos. De acuerdo a las autoras de esta ponencia, en los currículos de posgrado debe de reconocerse abiertamente la acción tutorial de los directores de tesis como una función indispensable para formar a los estudiantes en aspectos investigativos. Para ello se requiere que tanto los académicos, como los estudiantes acepten el desarrollar habilidades en cuanto a la comunicación, apertura y acompañamiento a lo largo de los programas educativos. 
Es importante que se promueva una formación en investigación en los estudiantes de posgrado, con el apoyo de su tutor (director de tesis) de tal manera que se vaya integrando un ethos considerado como capital científico por lo que se deben disponer de recursos institucionales para producir el conocimiento y estar en posibilidad de comunicar los hallazgos, fortaleciendo las LGACs, además de propiciar el establecer/consolidar redes académicas

Hay que sensibilizar a los estudiantes para que desarrollen el interés en su formación profesional integral y no sólo el ambicionar alcanzar el éxito escolar con un buen promedio que se pueda traducir en estímulos institucionales (De la Cruz, Chehaybar y Abreu, 2011).

La vinculación entre tutor-tutorado también favorece al primero en cuanto a su reconocimiento social, poder, estatus profesional y dinero ya que al reforzar la productividad conjunta (publicaciones científicas en las que se comparten el trabajo y los créditos), tiene acceso a la obtención de becas (el subir de nivel), el que aproveche colaboración, el que aumente su productividad anual (si aún no pertenece puede contribuir a que reúna lo necesario para ingresar al Sistema Nacional de Investigadores) o consiga financiamiento externo, entre otros beneficios. 


\section{REFERENCIAS}

ANUIES. (2011). Programas institucionales de tutorías. Una propuesta de la ANUIES. 3era. edición. México: ANUIES.

De la Cruz Flores, G., Chehaybar. E. y Abreu, L. F. Tutoria en educación superior: una revisión analítica a la bibliografía en Revista de la Educación Superior. Vol. XL (1), Núm. 157, enero - marzo de 2011.

Díaz Barriga, A. y Luna, A. B. Coordinadores. (2014). Metodología de la investigación educativa. México: Ediciones Díaz de Santos/Universidad Autónoma de Tlaxcala.

El Sahili, E. L. F. (2011). Docencia: Riesgos y desafíos. México: Trillas.

Flores, L., Vargas, J. y Domínguez, K. (2014). Plan de vida y carrera. México: Pearson.

Johnson, L., Adams Becker, S., Cummins, M., Estrada, V., Freeman, A., and Hall, C. (2016). NMC Horizon Report: 2016 Higher Education Edition. Austin, Texas: The New Media Consortium.

Ibarrola, M. y Anderson, L. W. Coordinadoras. (2015). La formación de nuevos investigadores educativos: diálogos y debates. México: ANUIES.

IPN. Un nuevo modelo educativo para el IPN. Materiales para la Reforma, Volumen 1. (2004). México: IPN.

Ley General de Educación. Diario Oficial de la Federación, 13 de julio de 1993. Revisada la última reforma de 01-06-2016. Recuperada de https://www.sep.gob.mx/work/models

/sep1/Resource/558c2c24-0b12-4676-ad90-8ab78086b184/ley_general_educacion.pdf

López, R. La tutoría como modalidad docente: de su condición artesanal a su escenario polifuncional en Ducoing, P. Coordinadora. (2010). Tutoría y mediación II. México: IISUE/UNAM.

Martínez, M., Retana, B. E y Sánchez, R. Identificación de las estrategias de regulación emocional del miedo en Adultos de la Ciudad de México en Psicología Iberoamericana. Vol. 17, Núm. 2, juliodiciembre 2009.

Martínez, R. (Julio-diciembre 2012). Quinta Hélice sistémica (QHS), un modelo para el desarrollo de políticas públicas en Revista Cooperativismo \& Desarrollo. Volumen 20, Núm. 101. Recuperado de: file:///C:/Users/julio/Downloads/Dialnet-QuintaHelice SistemicaGhsUnModeloPara ElDesarrolloDe4865365\%20(1).pdf.

Olea, E. (2016). El tutor como figura académica para el éxito en las unidades de aprendizaje. México: IPN.

Olivé, L. (2007). La ciencia y la tecnología en la edad del conocimiento. México: Fondo de Cultura Económica.

Programa de Desarrollo Institucional del IPN 2015-2018. Recuperado de http://www.ipn. mx/DG/Documents/PDI-2015-2018.pdf.

Reglamento General de Estudios. Gaceta Politécnica, Número extraordinario 866 del 13 de julio de 2011. 
Reglamento de Estudios de Posgrado del Instituto Politécnico Nacional. Gaceta Politécnica. Número extraordinario 633 del 31 de julio de 2006.

Reglamento de Estudios de Posgrado del Instituto Politécnico Nacional. Gaceta Politécnica. Número extraordinario 1358 del 15 de septiembre de 2017.

Resing, W., \& Drenth, P. (2007). Intelligence: knowing and measuring. Amsterdam: Publisher Nieuwezijds.

Sánchez, R. (2010). Enseñar a investigar. Una didáctica nueva de la investigación en ciencias sociales y humanas. México: IISUE/UNAM/ Plaza y Valdés Editores.

NOTA $^{\mathrm{i}}$ Conferencia magistral denominada Promoción de la Investigación; vínculos de las Redes y el COMIE, dictada por la Dra. Rosa Ma. Torres, presidenta del COMIE, el 22 de agosto de 2016, en la XXVII Jornada Académica de la Red Mexicana de Investigadores de la Investigación Educativa en San Luis Potosí.

NOTA $^{\text {ii }}$ El saber es lo que cada persona ha construido como resultado de sus estudios o de su experiencia y se establece como producto de la interacción entre conocimiento e información. El conocimiento es integrado por el sujeto a su haber, por lo que es propio, individual. La información es lo que se encuentra fuera del estudiante y el tutor y que está ahí para ser analizada y a la que todos tienen acceso.

NOTA $^{\text {iii }}$ Burnout proviene de las palabras burn out cuyo significado es estar quemado. Aplicado al agotamiento del profesor se entiende como una pérdida total de energía e interés que se traduce en la imposibilidad de funcionar efectivamente, resultado de la experiencia de demandas excesivas acerca de lo que se puede realizar o por una sobrecarga de trabajo durante un tiempo prolongado. 